\title{
Study on Autocatalytic Decomposition of Dimethyl Sulfoxide (DMSO)
}

Yoshikuni Deguchi $^{*},+, \S$, Masafumi Kono ${ }^{\sharp, \&}$, Yuto Koizumi $^{\S}$, Yu-ichiro Izato $^{\S}$, Atsumi Miyake $^{\S}$

${ }^{\dagger}$ Kaneka Corporation, 1-8, Miyamae-cho, Takasago-cho, Takasago Hyogo 676-8688, Japan

${ }^{\ddagger}$ Nippon Refine Co., Ltd, 2388-22, Yawatakaigandori, Ichihara, Chiba 290-0067, Japan

${ }^{\S}$ Yokohama National University, 79-7 Tokiwadai, Hodogaya-ku, Yokohama 240-8501, Japan

\section{Supporting Information}

Contents

1. Chemical Subjected for Analysis

2. Analytical Procedures

1) Apparatus

2) Analytical Conditions

3. Analytical Results

1) Gas Chromatogram

2) MS spectra and identification results 
1. Chemical Subjected for Analysis

Dimethyl Sulfoxide (DMSO) purchased from FUJIFILM Wako Pure Chemical Corporation, Japan.

2. Analytical Procedures

1) Apparatus

GC: Agilent Technologies 6890N

MS: Agilent Technologies 5975inert

2) Analytical Conditions

$\begin{array}{ll}\text { Column: } & \text { DB-WAX } 0.25 \mathrm{mmID} \\ \text { Oven Temperature: } & 40^{\circ} \mathrm{C}(5 \mathrm{~min})-20^{\circ} \mathrm{C} / \mathrm{min}-260^{\circ} \mathrm{C}(10 \mathrm{~min}) \\ \text { Injection Temperature: } & 200^{\circ} \mathrm{C} \\ \text { Carrier Gas: } & \mathrm{He}, 1 \mathrm{~mL} / \mathrm{min} \\ \text { Injection Method: } & \mathrm{Split} \text { with a ratio of } 1 / 20 \\ \text { Interface Temperature: } & 260^{\circ} \mathrm{C} \\ \text { Ionization Temperature: } & 250^{\circ} \mathrm{C} \\ \text { EM Voltage: } & 1,400 \mathrm{eV} \\ \text { Mass Range: } & \mathrm{m} / \mathrm{z} 29-800 \\ \text { Injection Volume: } & 1 \mu \mathrm{L}\end{array}$


3. Analytical Results

1) Peak Area Report

\begin{tabular}{|c|c|c|r|r|r|}
\hline Number & RT (min) & Identification & Peak Height & Peak Area & Area Ratio (\%) \\
\hline 1 & 10.4 & DMSO $^{\# 1)}$ & 56200457 & 20155140323 & 99.98 \\
\hline 2 & 11.0 & Unknown $^{\# 2)}$ & 176436 & 1480309 & 0.007 \\
\hline 3 & 11.6 & Unknown $^{\# 3)}$ & 116190 & 1204817 & 0.006 \\
\hline 4 & 12.1 & MSM $^{\# 4}$ & 93644 & 1218294 & 0.006 \\
\hline
\end{tabular}

Notes \#1 Dimethyl Sulfoxide

\#2 Unknown substance. MW: 92.

\#3 Unknown substance. MW: uncertain

\#4 Methylsulfonylmethane or Dimethyl Sulfone

2) Chromatograms and MS spectra

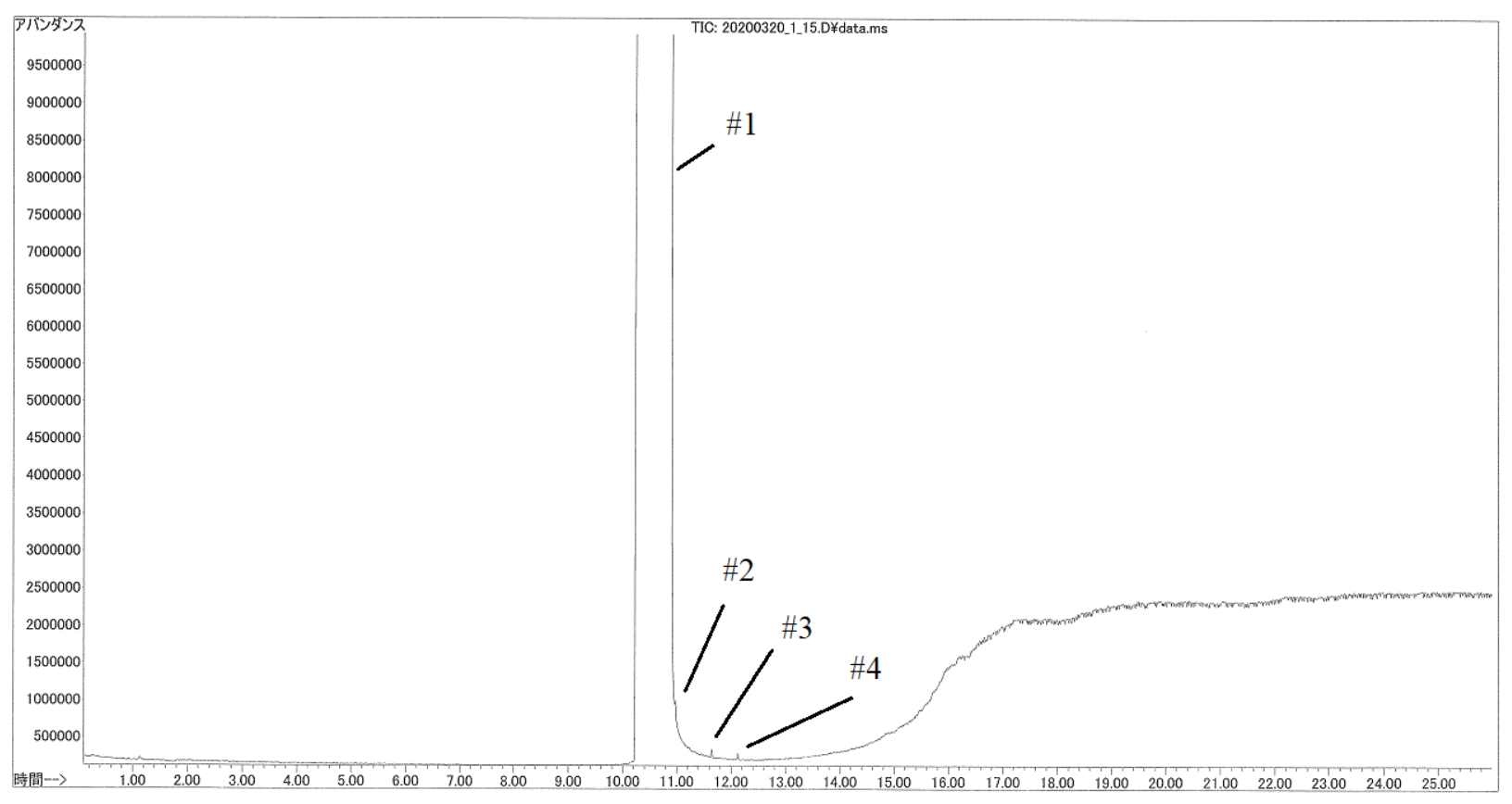

Figure SI1. GC Chromatogram 


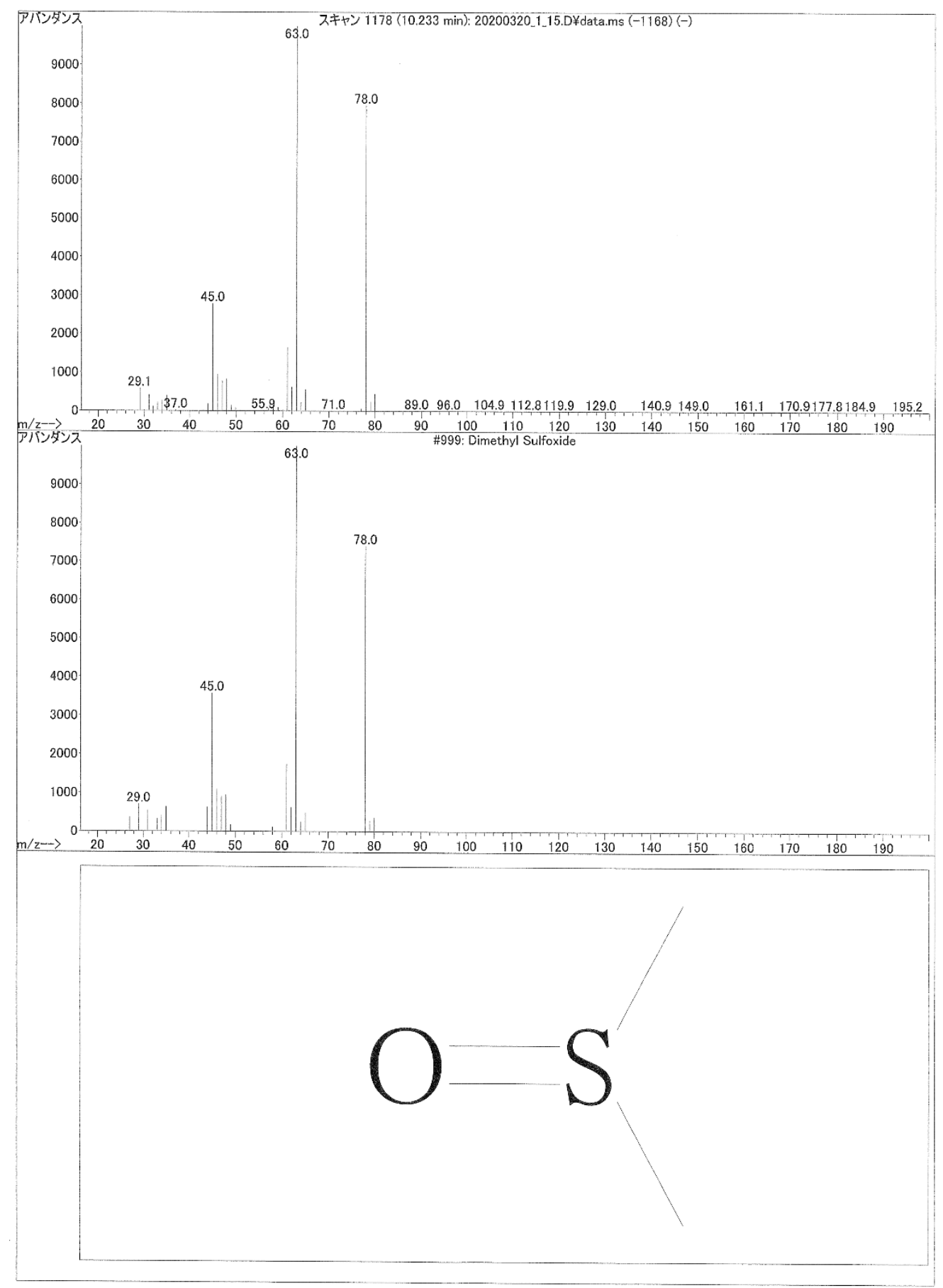

Figure SI2. Mass Spectrum of Peak \#1

Top: $\quad$ Mass spectrum obtained for Peak \#1

Middle: Library search result

Bottom: Identified chemical formula 


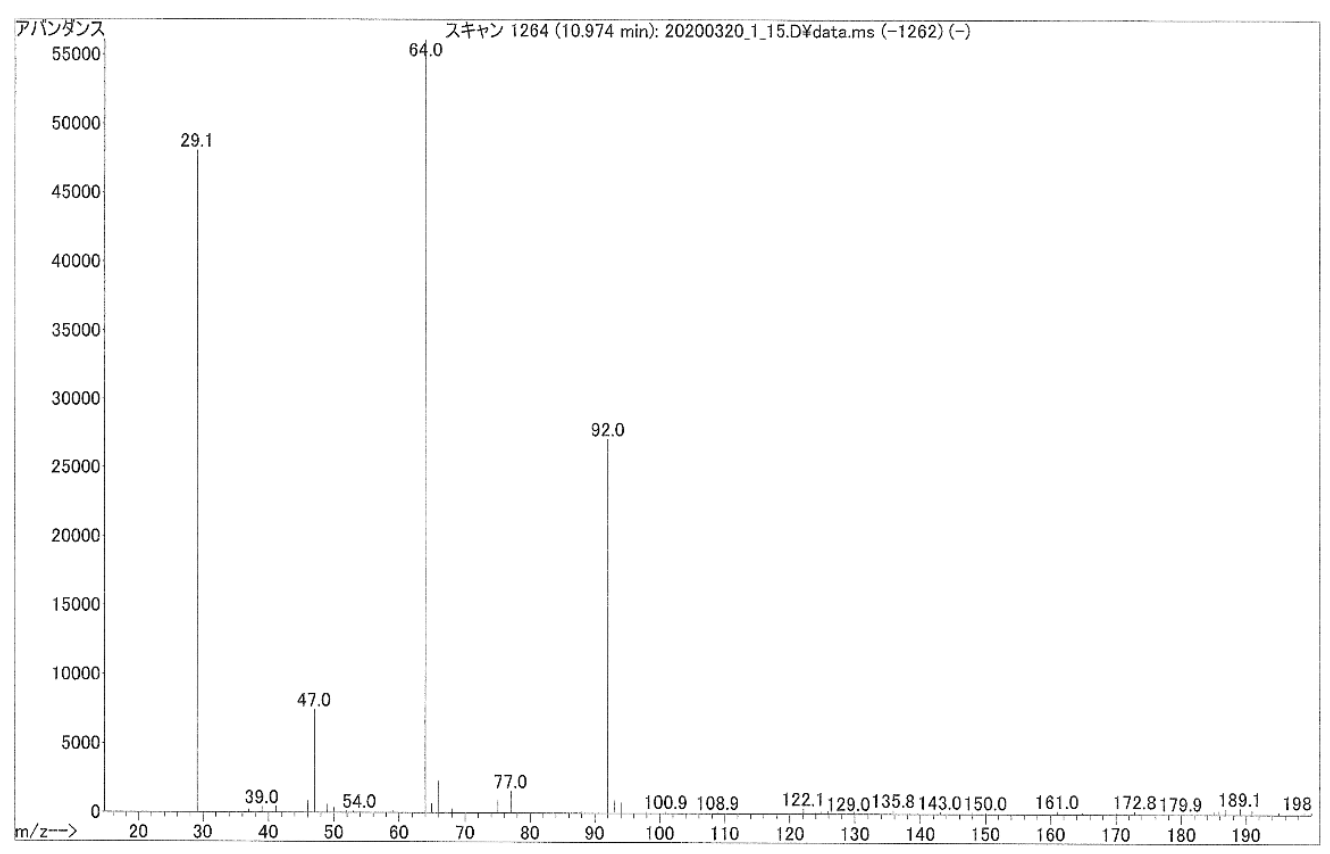

Figure SI3. Mass Spectrum of Peak \#2

Nothing matches in the library.

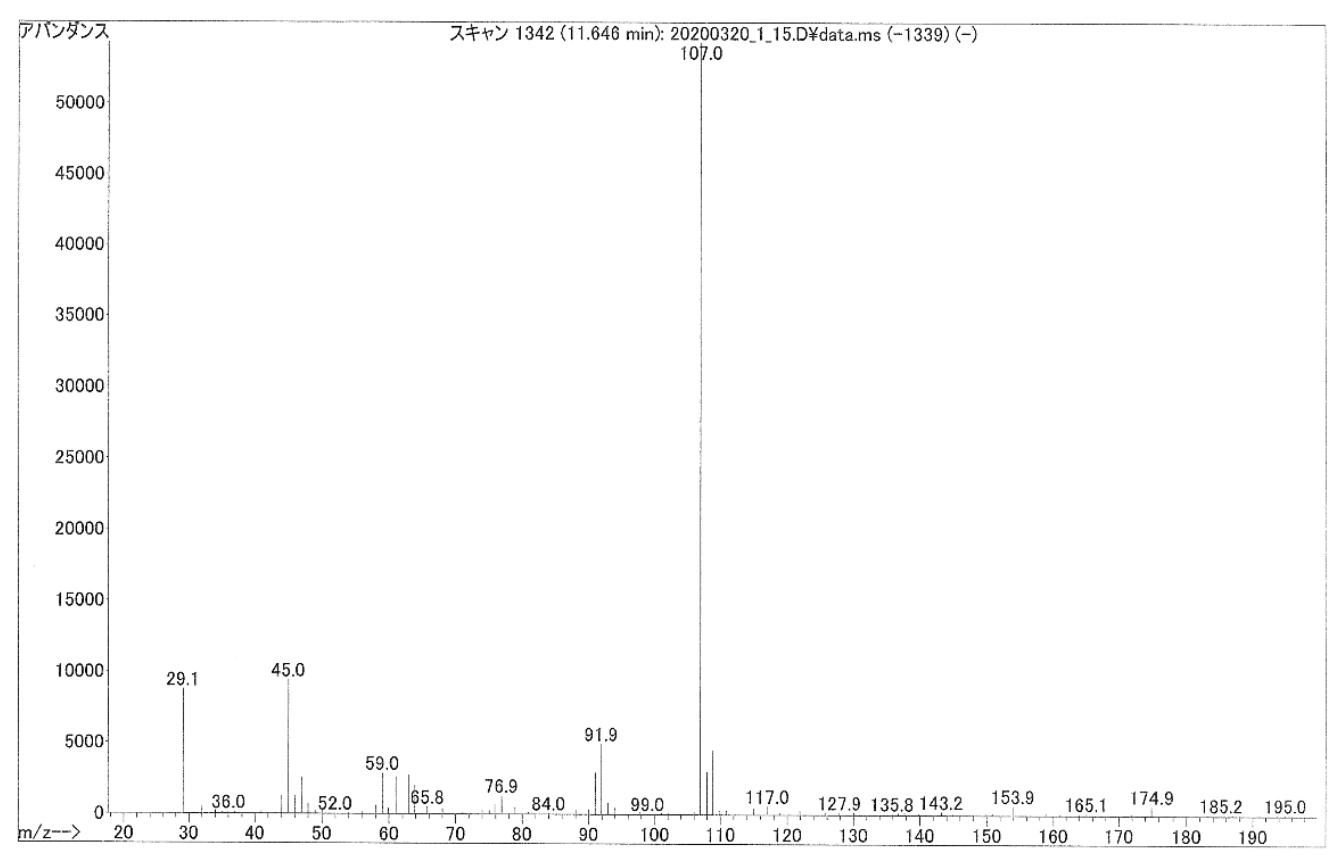

Figure SI4. Mass Spectrum of Peak \#3

Nothing matches in the library. 


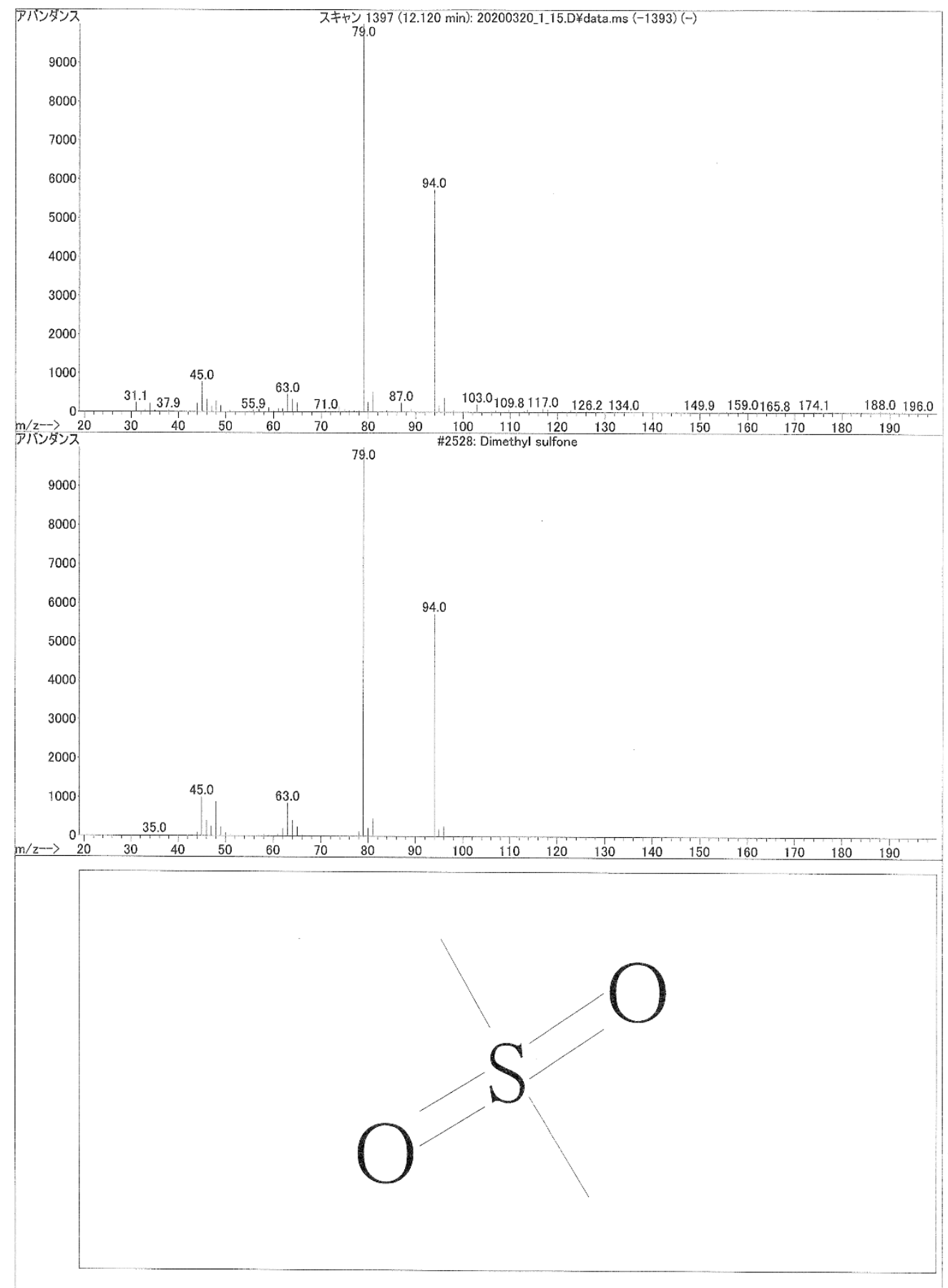

Figure SI5. Mass Spectrum of Peak \#4

Top: $\quad$ Mass spectrum obtained for Peak \#4

Middle: Library search result

Bottom: Identified chemical formula 